\title{
RESEARCH
}

Open Access

\section{Effectiveness comparison of indocyanine green retention test with the cirrhotic severity scoring in evaluating the pathological severity of liver cirrhosis in patients with hepatocellular carcinoma and Child-Pugh grade $A$ liver function}

Jin Gu, Erlei Zhang, Binyong Liang, Zunyi Zhang, Xiaoping Chen and Zhiyong Huang*

\begin{abstract}
Background: Evaluating cirrhotic severity is essential for individualizing surgical modalities for patients with hepatocellular carcinoma (HCC). Our previous study proposed a non-invasive method named cirrhotic severity scoring (CSS) to stage liver cirrhosis. Indocyanine green retention rate at 15 min (ICG-R15) has been widely used for the preoperative evaluation of hepatic functional reserve; however, whether ICG-R15 is well correlated with cirrhotic severity, and especially whether comparable with CSS in predicting cirrhotic severity in HCC patients with ChildPugh grade A liver function remains unknown.

Methods: Overall, $510 \mathrm{HCC}$ patients with Child-Pugh grade A liver function undergoing hepatectomy between January 2011 and December 2014 were retrospectively studied. Cirrhotic severity was pathologically assessed using the Laennec staging system. The correlations between ICG-R15, CSS, and cirrhotic severity were analyzed. Furthermore, the performance of ICG-R15 and CSS in predicting posthepatectomy liver failure (PHLF) and 90-day mortality was compared.

Results: Patients with no, mild, moderate, and severe cirrhosis accounted for 15.9\%, 29.2\%, 35.9\%, and 19.0\%, respectively, in the entire cohort. ICG-R15 was found to be less than $10 \%$ in $100 \%, 93.3 \%, 86.3 \%$, and $70.1 \%$ of the patients with no, mild, moderate, and severe cirrhosis, respectively. There was only a weak correlation between ICG-R15 and the pathological severity of liver cirrhosis $(r=0.325 ; P<0.001)$. However, CSS showed a strong correlation with the pathological severity of liver cirrhosis $(r=0.788 ; P<0.001)$. For those with ICG-R15 in the normal range, the accuracy of CSS in diagnosing no/mild, moderate, and severe cirrhosis was $89.1 \%, 72.8 \%$, and $72.1 \%$, respectively. In addition, CSS was superior to ICG-R15 in predicting PHLF and 90-day mortality.

(Continued on next page)
\end{abstract}

* Correspondence: zyhuang126@126.com

Hepatic Surgery Center, Tongji Hospital, Tongji Medical College, Huazhong

University of Science and Technology, 1095 Jie Fang Da Dao, Wuhan, China

(c) The Author(s). 2020 Open Access This article is licensed under a Creative Commons Attribution 4.0 International License, which permits use, sharing, adaptation, distribution and reproduction in any medium or format, as long as you give appropriate credit to the original author(s) and the source, provide a link to the Creative Commons licence, and indicate if changes were made. The images or other third party material in this article are included in the article's Creative Commons licence, unless indicated otherwise in a credit line to the material. If material is not included in the article's Creative Commons licence and your intended use is not permitted by statutory regulation or exceeds the permitted use, you will need to obtain permission directly from the copyright holder. To view a copy of this licence, visit http://creativecommons.org/licenses/by/4.0/. The Creative Commons Public Domain Dedication waiver (http://creativecommons.org/publicdomain/zero/1.0/) applies to the data made available in this article, unless otherwise stated in a credit line to the data. 
(Continued from previous page)

Conclusions: CSS was more useful than ICG-R15 in the preoperative assessment of cirrhotic severity in HCC patients with Child-Pugh grade A liver function. More studies are needed to further validate CSS in patients with different ChildPugh grades.

Keywords: Hepatocellular carcinoma, Liver cirrhosis, Laennec staging system, Indocyanine green retention test, Cirrhotic severity scoring, Posthepatectomy liver failure, Mortality

\section{Introduction}

Hepatocellular carcinoma (HCC) is the sixth most common malignancy and the fourth leading cause of cancerrelated death in the world [1]. Hepatectomy remains the mainstay curative option for HCC patients [2]. However, in China, more than $80 \%$ of patients develop HCC in the background of cirrhosis [3]. Posthepatectomy liver failure (PHLF) resulting from the insufficient residual liver function is one of the most serious complications for HCC patients undergoing hepatic resection $[4,5]$, and the incidence of PHLF is significantly associated with cirrhotic severity [6]. Therefore, preoperative evaluation of cirrhotic severity is of significant importance before making a surgical treatment strategy.

Indocyanine green (ICG) is a water-soluble anionic compound that mainly binds to plasma proteins after intravenous administration. It is selectively taken up by hepatocytes and excreted unchanged into the bile. It is not metabolized and does not enter the enterohepatic circulation [7]. The clearance of ICG relies on the function of hepatocytes, biliary excretion, and liver blood flow $[8,9]$. ICG retention rate at $15 \mathrm{~min}$ (ICG-R15) is a widely used clinical parameter calculated from liver clearance of ICG. Many studies have indicated that ICGR15 could serve as a quantitative parameter in assessing liver functional reserve [10, 11]. However, whether ICGR15 is effective in evaluating cirrhotic severity in HCC patients with Child-Pugh grade A liver function has never been elucidated.

In the assessment of cirrhotic severity, liver biopsy is still regarded as the reference standard. Nevertheless, it is an invasive procedure, and its results are influenced by several factors, such as inter-observer variability and sampling errors $[12,13]$. Thus, liver biopsy is not recommended to be performed in the preoperative evaluation of cirrhotic severity. The cirrhotic severity scoring (CSS) was previously developed by our team as a non-invasive method to stage liver cirrhosis in HCC patients [14]. It relies on the patient's portal vein diameter, splenic thickness, platelet count, and varicosity, which are routinely measured and available for virtually all patients with HCC scheduled for hepatectomy. This method has exhibited a high degree of diagnostic accuracy in predicting the pathological severity of liver cirrhosis; however, whether CSS is superior to ICG-R15 in assessing cirrhotic severity remains unexplored.
The present study aimed to investigate the effectiveness of ICG-R15 in the preoperative evaluation of cirrhotic severity in HCC patients with Child-Pugh grade A liver function and compare its diagnostic performance with that of CSS. Furthermore, the performance of ICGR15 and CSS in predicting PHLF and 90-day mortality was compared.

\section{Patients and methods \\ Patients}

This study was conducted in accordance with the standards of the Declaration of Helsinki and approved by the medical ethics committee of Tongji Hospital, Huazhong University of Science and Technology, China. Written informed consent for hepatectomy and further research was obtained from all patients. A total of $510 \mathrm{HCC}$ patients with Child-Pugh grade A liver function who underwent hepatic resection at the Hepatic Surgery Center of Tongji Hospital between January 2011 and December 2014 were retrospectively analyzed. All patients received routine blood tests, chest X-ray, abdominal ultrasonography, upper gastrointestinal endoscopy, computed tomography, and ICG-R15 for preoperative assessment. Clinicopathological records of all patients were obtained from the computerized database maintained by Tongji Hospital. The grades of esophageal varices were classified as follows: mild, straight varices not disappearing with insufflations; moderate, enlarged tortuous, occupying less than $1 / 3$ of the lumen; and severe, coil-shaped, occupying more than $1 / 3$ of the lumen [15]. Major resection was defined as resection of 3 or more Couinaud liver segments [16]. PHLF was defined by an increased international normalized ratio and concomitant hyperbilirubinaemia on or after postoperative day 5 as proposed by the International Study Group of Liver Surgery [4]. Postoperative mortality was defined as death within 90 days after hepatectomy.

\section{ICG retention test}

ICG retention test was carried out by injecting a dose of $0.5 \mathrm{mg} / \mathrm{kg}$ of ICG rapidly via a peripheral vein of the forearm. A nasal mucosa probe was employed with the purpose of monitoring changes in ICG concentrations. The value of ICG-R15 was calculated by a Pulse Dye Densito-Graph Analyzer (DDG-3300 K, Nihon Kohden, Tokyo, Japan). 


\section{Pathological assessment of cirrhotic severity}

The pathological severity of liver cirrhosis in the nontumorous tissue of the resected liver specimens was evaluated by two experienced pathologists who were blinded to the clinical information according to the Laennec staging system. Cirrhotic severity was staged as follows: no cirrhosis (combination of F0-F3); mild cirrhosis (F4A, marked septation with rounded contours); moderate cirrhosis (F4B, at least 2 broad septa); and severe cirrhosis ( $\mathrm{F} 4 \mathrm{C}$, at least 1 very broad septum or many minute nodules) [17].

\section{Cirrhotic severity scoring (CSS) for evaluating cirrhotic severity}

The calculation of CSS was based on the portal vein diameter, splenic thickness, platelet count, and varicosity as described in Table 1 [14].

\section{Statistical analysis}

Continuous variables were expressed as mean \pm standard deviation. Categorical variables were expressed as numbers and percentages and compared using Fisher's exact test. Spearman correlation coefficients were used to analyze the correlations between ICG-R15, CSS, and cirrhotic severity, and correlation coefficients were compared using Fisher $Z$ test. The predictive ability of ICGR15 and CSS was assessed using receiver operating characteristic (ROC) curves. Areas under the ROC curves (AUCs) were compared using the Delong method [18]. $P$ $<0.05$ was considered statistically significant. Statistical analyses were performed using the SPSS version 20.0 for Windows (SPSS Inc, Chicago, IL, USA) and Medcalc version 18.2.1 (MedCalc Software bvba, Mariakerke, Belgium).

\section{Results}

\section{Patients' characteristics}

The characteristics of all patients are summarized in Table 2. There were 449 men and 61 women, and the mean age was $50.1 \pm 11.2$ years. Hepatitis B was the main etiology (89.6\%) followed by hepatitis C (2.0\%). The mean value of ICG-R15 was $5.7 \pm 4.6 \%$. The mean tumor diameter was $6.6 \pm 3.7 \mathrm{~cm}$ (range, $1.0-20.0 \mathrm{~cm}$ ). Four hundred and forty-seven patients (87.6\%) had a single tumor, and $63(12.4 \%)$ had 2 or more tumors. Surgical resections included 92 major resections and 418 minor resections. Based on the Laennec staging system, 81 patients (15.9\%) were without cirrhosis (F0-F3), whereas 149 (29.2\%) were diagnosed with mild cirrhosis (F4A), 183 (35.9\%) with moderate cirrhosis (F4B), and 97 (19.0\%) with severe cirrhosis (F4C). Representative figures of the corresponding Laennec stage are exhibited in Fig. 1.

\section{Relationship between ICG-R15 and the Laennec stage}

Although a positive correlation was observed between ICG-R15 and the Laennec stage in the entire cohort, the Spearman correlation coefficient was only $0.325(P<$ 0.001; Fig. 2a), which reflected a very weak relationship. The number of patients with different levels of ICG-R15 in the corresponding pathological stage of liver cirrhosis is shown in Fig. 2b. In the no cirrhosis (F0-F3) group, all patients (100\%) had ICG-R15 values below 10\%. In the mild cirrhosis (F4A) group, 139 patients (93.3\%) had ICG-R15 values below 10\%, and 10 (6.7\%) had ICG-R15 values between $10 \%$ and $19 \%$. In the moderate cirrhosis (F4B) group, 158 patients (86.3\%) had ICG-R15 values below $10 \%$, and 25 (13.7\%) had ICG-R15 values between 10 and 19\%. In the severe cirrhosis (F4C) group, 68 patients (70.1\%) had ICG-R15 values below 10\%, 23 (23.7\%) had ICG-R15 values between 10 and 19\%, and 6 (6.2\%) had ICG-R15 values $\geq 20 \%$.

\section{CSS is significantly superior to ICG-R15 in evaluating cirrhotic severity}

CSS showed a strong correlation with the Laennec stage $(r=0.788 ; P<0.001$; Fig. 3). The Spearman correlation coefficient for CSS was significantly higher than that for ICG-R15 $(P<0.001)$. The diagnostic performance of ICG-R15 and CSS in the entire cohort is shown in Fig. 4. ROC analysis revealed that CSS exhibited a better performance than ICG-R15 in the diagnosis of moderate (AUC $=0.896$ vs $0.663 ; P<0.001$ ) and severe cirrhosis (AUC $=0.931$ vs $0.707 ; P<0.001)$.

Table 1 Assessment of cirrhotic severity according to the cirrhotic severity scoring

\begin{tabular}{|c|c|c|c|}
\hline \multirow[t]{2}{*}{ Variables } & \multicolumn{3}{|l|}{ Score } \\
\hline & 0 & 1 & 2 \\
\hline Portal vein diameter $(\mathrm{cm})$ & $<1.2$ & $1.2-1.4$ & $>1.4$ \\
\hline Splenic thickness (cm) & $<4.0$ & $4.0-5.0$ & $>5.0$ \\
\hline Platelet count $\left(10^{9} / \mathrm{L}\right)$ & $\geq 100$ & $70-100$ & $<70$ \\
\hline \multirow[t]{2}{*}{ Varicosity } & No & Mild & Moderate/severe \\
\hline & No/mild cirrhosis & Moderate cirrhosis & Severe cirrhosis \\
\hline CSS & $0-1$ & $2-3$ & $\geq 4$ \\
\hline
\end{tabular}

CSS cirrhotic severity scoring 
Table 2 Baseline characteristics and postoperative short-term outcomes of the study population

\begin{tabular}{|c|c|}
\hline Variables & Patients $(n=510)$ \\
\hline Age (years) & $50.1 \pm 11.2$ \\
\hline \multicolumn{2}{|l|}{ Sex, $n(\%)$} \\
\hline Men & $449(88.0 \%)$ \\
\hline Women & $61(12.0 \%)$ \\
\hline \multicolumn{2}{|l|}{ Etiology, $n(\%)$} \\
\hline Hepatitis B & $457(89.6 \%)$ \\
\hline Hepatitis C & $10(2.0 \%)$ \\
\hline Other & $43(8.4 \%)$ \\
\hline ALT (U/L) & $36.5 \pm 24.6$ \\
\hline AST (U/L) & $39.2 \pm 21.2$ \\
\hline Albumin $(g / L)$ & $39.7 \pm 4.5$ \\
\hline Total bilirubin ( $\mu \mathrm{mol} / \mathrm{L})$ & $13.4 \pm 5.2$ \\
\hline Creatinine $(\mu \mathrm{mol} / \mathrm{L})$ & $70.5 \pm 15.2$ \\
\hline Platelet count $\left(10^{9} / \mathrm{L}\right)$ & $143.6 \pm 72.5$ \\
\hline INR & $1.1 \pm 0.1$ \\
\hline PT (s) & $13.1 \pm 1.6$ \\
\hline ICG-R15 (\%) & $5.7 \pm 4.6$ \\
\hline Tumor size $(\mathrm{cm})$ & $6.6 \pm 3.7$ \\
\hline \multicolumn{2}{|l|}{ Tumor number, $n$ (\%) } \\
\hline 1 & $447(87.6 \%)$ \\
\hline$\geq 2$ & $63(12.4 \%)$ \\
\hline Portal vein diameter $(\mathrm{cm})$ & $1.2 \pm 0.2$ \\
\hline Splenic thickness (cm) & $4.0 \pm 0.8$ \\
\hline \multicolumn{2}{|l|}{ Varicosity, $n(\%)$} \\
\hline No & $437(85.7 \%)$ \\
\hline Mild & $43(8.4 \%)$ \\
\hline Moderate & $13(2.5 \%)$ \\
\hline Severe & $17(3.3 \%)$ \\
\hline \multicolumn{2}{|l|}{$\operatorname{CSS}, n(\%)$} \\
\hline No/mild cirrhosis & $246(48.2 \%)$ \\
\hline Moderate cirrhosis & $169(33.1 \%)$ \\
\hline Severe cirrhosis & $95(18.6 \%)$ \\
\hline \multicolumn{2}{|l|}{ Extent of hepatectomy, n (\%) } \\
\hline Major resection & $92(18.0 \%)$ \\
\hline Minor resection & $418(82.0 \%)$ \\
\hline Intraoperative blood loss (ml) & $379.6 \pm 378.9$ \\
\hline Intraoperative RBC transfusion, $n(\%)$ & $88(17.3 \%)$ \\
\hline \multicolumn{2}{|l|}{ Pathological stage, $n(\%)$} \\
\hline FO-F3 & $81(15.9 \%)$ \\
\hline F4A & $149(29.2 \%)$ \\
\hline$F 4 B$ & $183(35.9 \%)$ \\
\hline F4C & $97(19.0 \%)$ \\
\hline PHLF, $n(\%)$ & $59(11.6 \%)$ \\
\hline 90-day mortality, n (\%) & $9(1.8 \%)$ \\
\hline
\end{tabular}

Data are expressed either as mean \pm standard deviation for continuous variables or as number of patients with percentages in parentheses for categorical variables ALT alanine aminotransferase; AST aspartate aminotransferase; INR international normalized ratio; PT prothrombin time; ICG-R15 indocyanine green retention rate at 15 min; CSS cirrhotic severity scoring; $R B C$ red blood cell; PHLF posthepatectomy liver failure
Diagnostic accuracy of CSS in discriminating cirrhotic severity in patients with a normal ICG-R15 value

We further assessed the discriminative ability of CSS for cirrhotic severity in 446 patients with ICG-R15 in the normal range. The proportions of patients with Laennec stage F0-F3, F4A, F4B, and F4C were 18.2\%, 31.2\%, $35.4 \%$, and $15.2 \%$, respectively. According to the CSS, 233 patients $(52.2 \%)$ had no/mild cirrhosis, 155 (34.8\%) had moderate cirrhosis, and 58 (13.0\%) had severe cirrhosis. The CSS was validated by comparison with the pathologically confirmed cirrhotic stage (Laennec stage). CSS was correct in $89.1 \%$ of patients with Laennec stage F0-F4A $(n=220), 72.8 \%$ with F4B $(n=158)$, and $72.1 \%$ with F4C $(n=68)$. Furthermore, ROC analysis showed that the AUCs of CSS in the diagnosis of moderate and severe cirrhosis in patients with a normal ICG-R15 value were 0.897 and 0.929 , respectively.

\section{Performance of ICG-R15 and CSS in predicting PHLF and mortality}

A total of 59 patients (11.6\%) developed PHLF (Table 2). Using ROC analysis, CSS was found to have a greater ability to predict PHLF compared with ICG-R15 (Fig. 5a). The AUC of CSS in predicting PHLF was 0.772, which was significantly higher than that of ICG-R15 (AUC = $0.657 ; P=0.004)$.

The incidence of 90-day mortality was $1.8 \%(n=9)$. Eight patients died of PHLF, and the remaining 1 patient died of acute respiratory distress syndrome. The 90-day mortality rate in patients with PHLF was $13.6 \%$, which was significantly higher than that in patients without PHLF (0.2\%; $P<0.001)$. ROC analysis showed that CSS was superior to ICG-R15 in predicting 90-day mortality (Fig. 5b). The AUC of CSS in predicting 90-day mortality was 0.722 , which was higher than that of ICG-R15 (AUC $=0.640)$, although the difference was not statistically significant $(P=0.379)$.

\section{Discussion}

ICG-R15 test is a well-established method to assess liver functional reserve. Based on the levels of ICG-R15, a decision tree was proposed for guiding the safe extent of hepatectomy, and ICG-R15 test was regarded as the most determining factor in this decision tree [19]. For example, trisectorectomy or bisectorectomy can be allowed in patients with an ICG-R15 value below $10 \%$. However, this algorithm did not take cirrhotic severity into consideration. The present study indicated that in 446 patients with ICG-R15 in the normal range, the proportion of patients with moderate or severe cirrhosis was as high as $50.7 \%$. Under this circumstance, it would not be appropriate to determine the extent of hepatectomy only based on the ICG-R15 value. Cirrhotic severity cannot be neglected when making surgical decisions. 

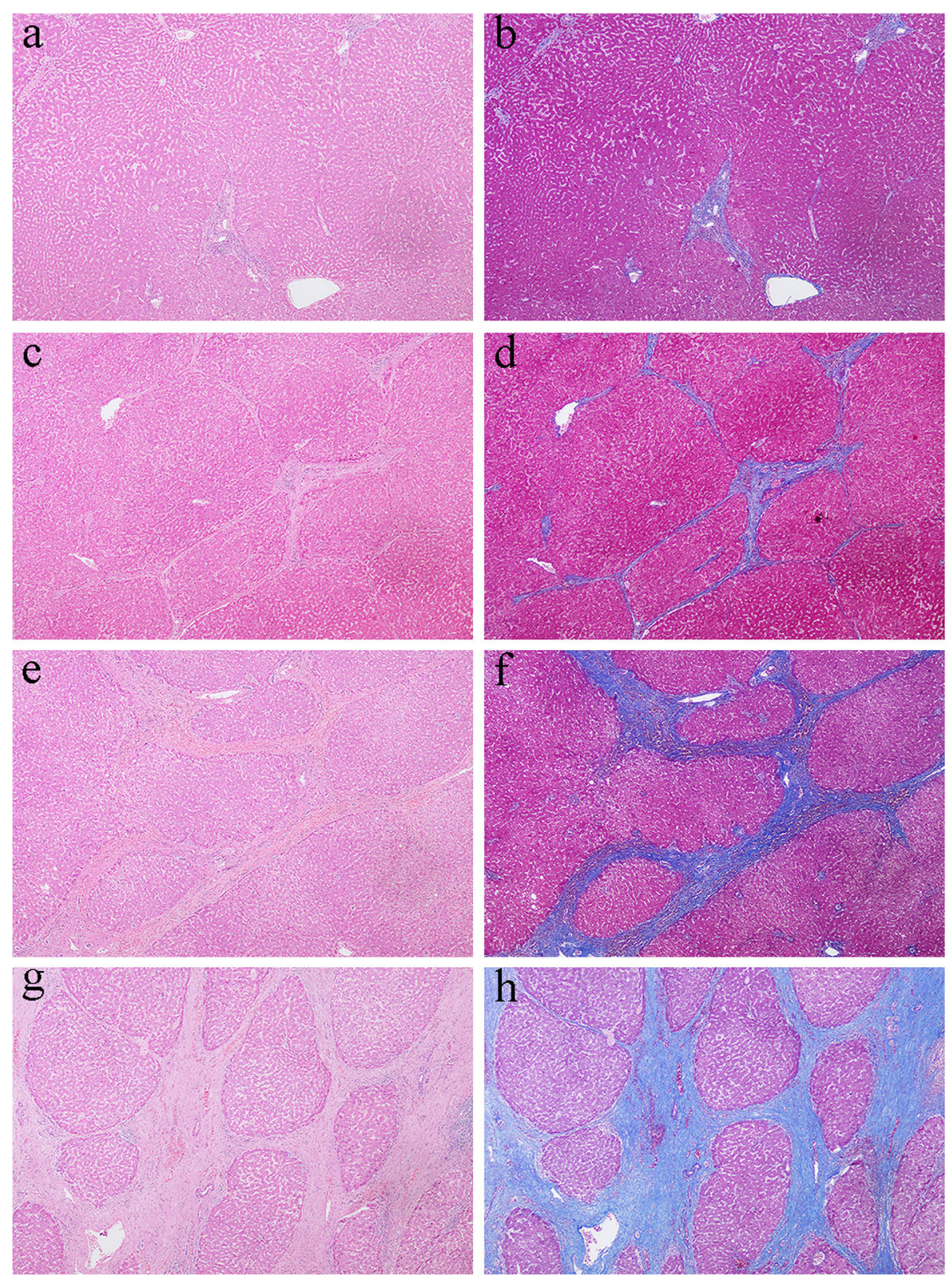

Fig. 1 Pathological sub-classification of liver cirrhosis according to the Laennec staging system. a, b Show no cirrhosis (combination of F0-F3) ( $H \& E$ and Masson trichrome stain, respectively, $\times 40$ ). c, d Show mild cirrhosis (F4A) (H\&E and Masson trichrome stain, respectively, $\times 40)$. e, $\mathbf{f}$ Show moderate cirrhosis (F4B) (H\&E and Masson trichrome stain, respectively, $\times 40$ ). $\mathbf{g}, \mathbf{h}$ Show severe cirrhosis (F4C) (H\&E and Masson trichrome stain, respectively, $\times 40)$. H\&E, hematoxylin-eosin

A study conducted by Ishikawa in 139 HCC patients undergoing hepatic resection over a 10 -year period revealed that both liver cirrhosis and ICG-R15 had a marked effect on the incidence of postoperative complications [20]. Our previous research also demonstrated that the incidence of PHLF was $38.1 \%$ in patients with moderate cirrhosis after resection of 3 or more liver segments and $63.2 \%$ in those with severe cirrhosis after resection of 2 or more liver segments. Thus, only minor resection could be recommended to patients with moderate or severe cirrhosis [6].

Recently, Moller and colleagues have evaluated the retention rate of ICG in both healthy subjects and cirrhotic patients with different Child-Pugh grades and found that ICG-R15 was higher in cirrhotic patients than in healthy subjects and increased with the increment of Child-Pugh grades [21]. Lau et al. [22] also found that ICG-R15 in HCC patients with cirrhosis or chronic 

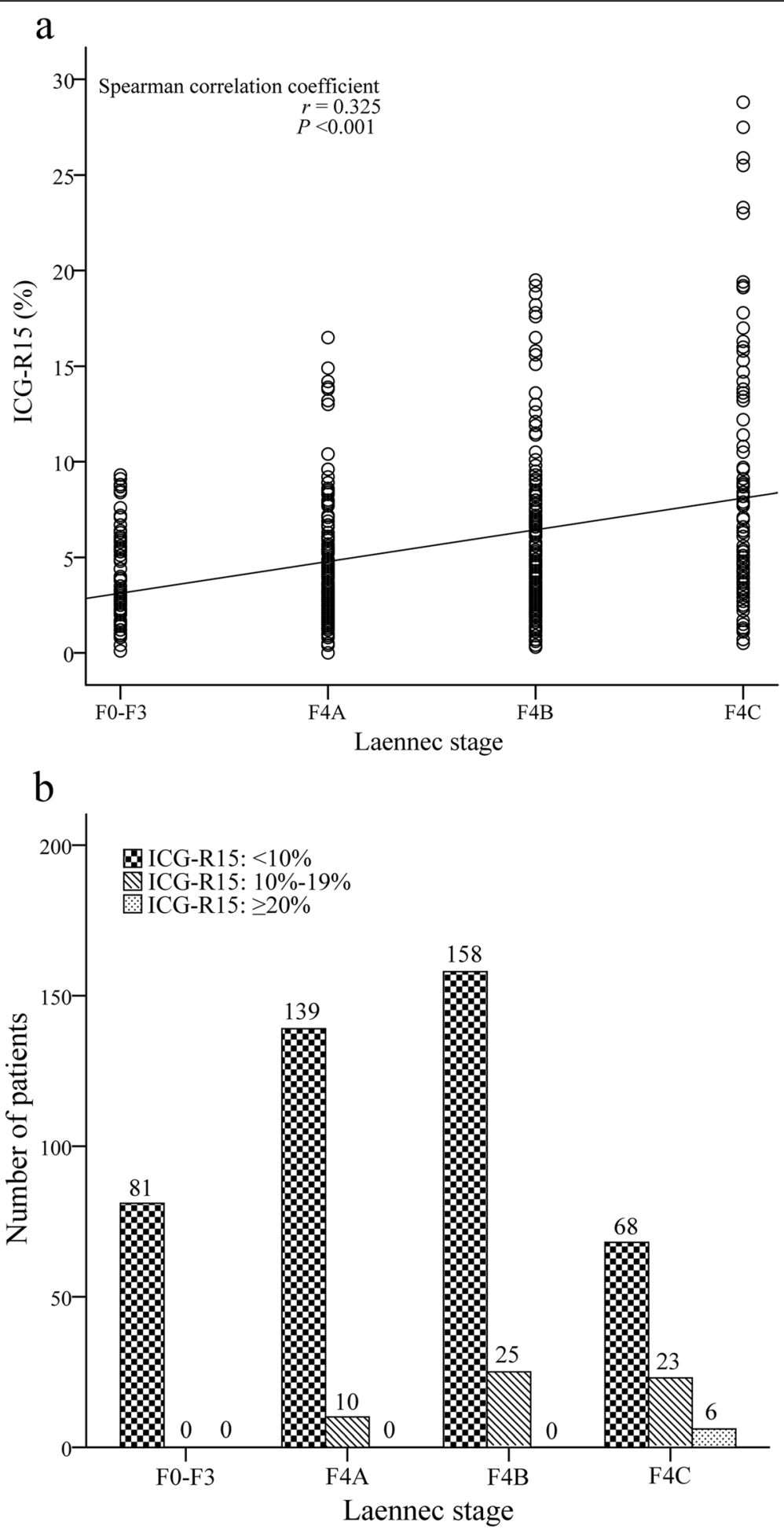

Fig. 2 a Correlation between ICG-R15 and the Laennec stage. b The bar chart indicates the pattern of ICG-R15 distribution in the corresponding pathological stage of liver cirrhosis. ICG-R15, indocyanine green retention rate at $15 \mathrm{~min}$

active hepatitis was higher than that in patients with normal liver. It was suggested that intrahepatic vascular shunts and sinusoidal capillarization might be the main reasons why ICG-R15 was increased in patients with cirrhosis [11]. However, whether ICG-R15 is helpful in predicting the pathological severity of liver cirrhosis in HCC patients with Child-Pugh grade A liver function remains unknown. To the best of our knowledge, the 


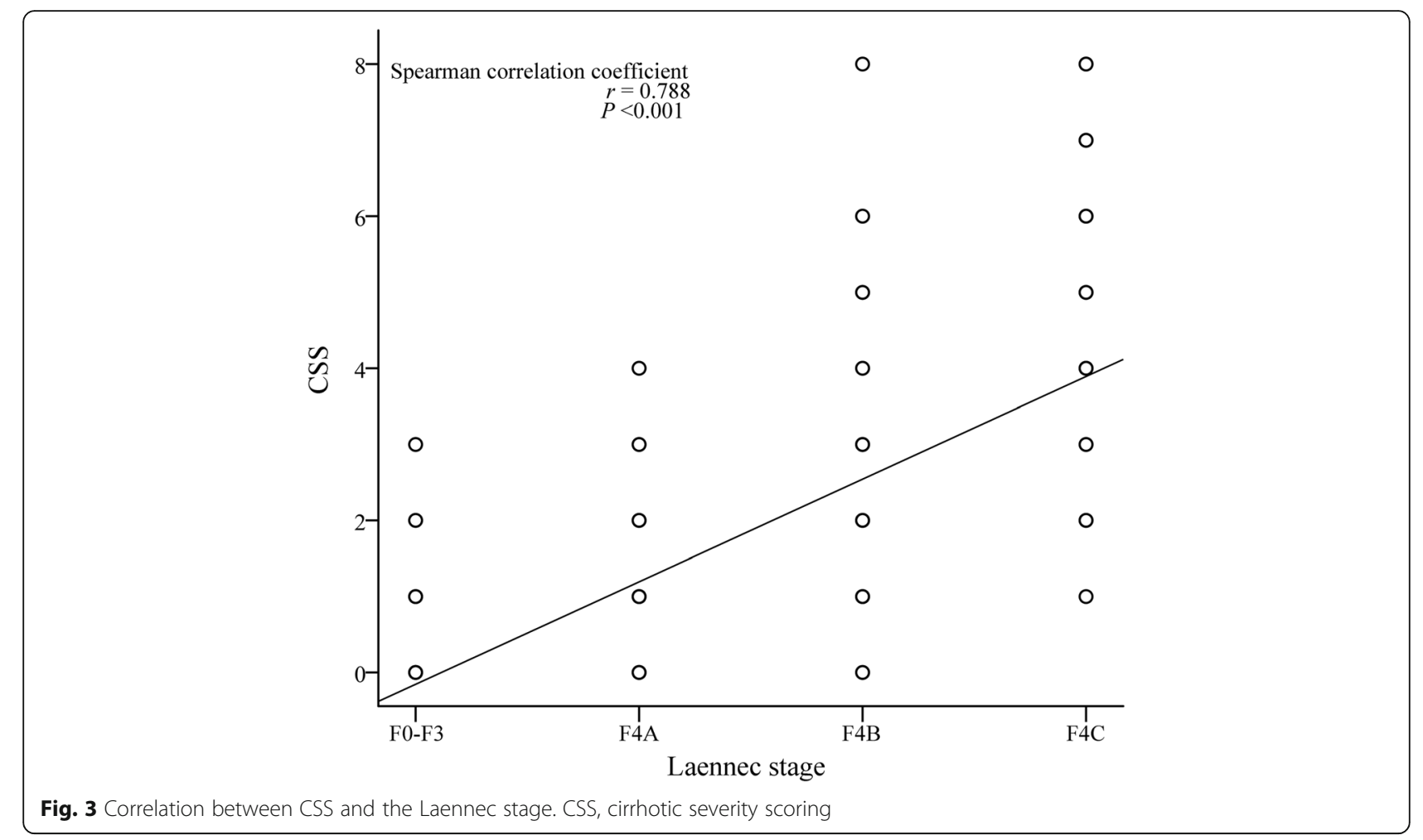

present study was the first in this regard. In this study, liver cirrhosis was pathologically staged according to the Laennec staging system. Although ICG-R15 was increased with more severe liver cirrhosis in HCC patients with Child-Pugh grade A liver function, correlation analysis revealed that ICG-R15 was weakly correlated with the Laennec stage $(r=0.325 ; P<0.001)$. Besides, the majority of patients in the corresponding cirrhotic stage had an ICG-R15 value below 10\%. ROC analysis further showed that the AUCs of ICG-R15 in the diagnosis of moderate and severe cirrhosis were only 0.663 and 0.707 , respectively. These results indicated that ICG-R15 was not reliable in assessing cirrhotic severity in HCC patients with Child-Pugh grade A liver function.

In recent years, several non-invasive methods based on serologic tests and imaging techniques have been developed to evaluate liver fibrosis, including aspartate aminotransferase-platelet ratio index (APRI) [23], fibrosis index based on the four factors (FIB-4) [24], King's score [25], and transient elastography (TE, FibroScan) [26]. However, these methods were employed to assess the severity of liver fibrosis rather than cirrhosis. It is unclear whether they are effective in evaluating cirrhotic severity in HCC patients. In 2016, we proposed a noninvasive method named CSS to predict cirrhotic severity [14]. The CSS is based on clinical parameters that are easily obtained through preoperative examinations. In this study, we demonstrated that CSS was significantly correlated with the pathological severity of liver cirrhosis in HCC patients with Child-Pugh grade A liver function $(r=0.788 ; P<0.001)$, consistent with previous findings [14]. In addition, we compared the efficacy of ICG-R15 with CSS in assessing cirrhotic severity and found that CSS showed a stronger correlation with the pathological severity of liver cirrhosis than did ICG-R15 and a better performance than ICG-R15 in the prediction of moderate and severe cirrhosis. Our study further revealed that in patients with a normal ICG-R15 value, the accuracy of CSS in diagnosing no/mild, moderate, and severe cirrhosis was $89.09 \%, 72.78 \%$, and $72.06 \%$, respectively. Therefore, CSS could function as a useful non-invasive method to assess cirrhotic severity even in HCC patients with an ICG-R15 value below 10\%.

PHLF is a dreadful complication after hepatectomy and remains the main cause of perioperative death [4-6]. In the present study, $11.6 \%$ of patients developed PHLF. The 90-day mortality rate in patients with PHLF was up to $13.6 \%$, whereas that in patients without PHLF was only $0.2 \%$. ROC analysis revealed that the AUCs of CSS in predicting PHLF and 90-day mortality were higher than those of ICG-R15, indicating that CSS had a better predictive value for PHLF and 90-day mortality than ICG-R15.

Several limitations exist in this study. First, this was a retrospective study, thus selection bias could occur. Second, the majority of HCC patients in this study were infected by hepatitis B virus, which is different from patients infected by hepatitis $\mathrm{C}$ virus in most Western countries or Japan. Third, our study took place in a 


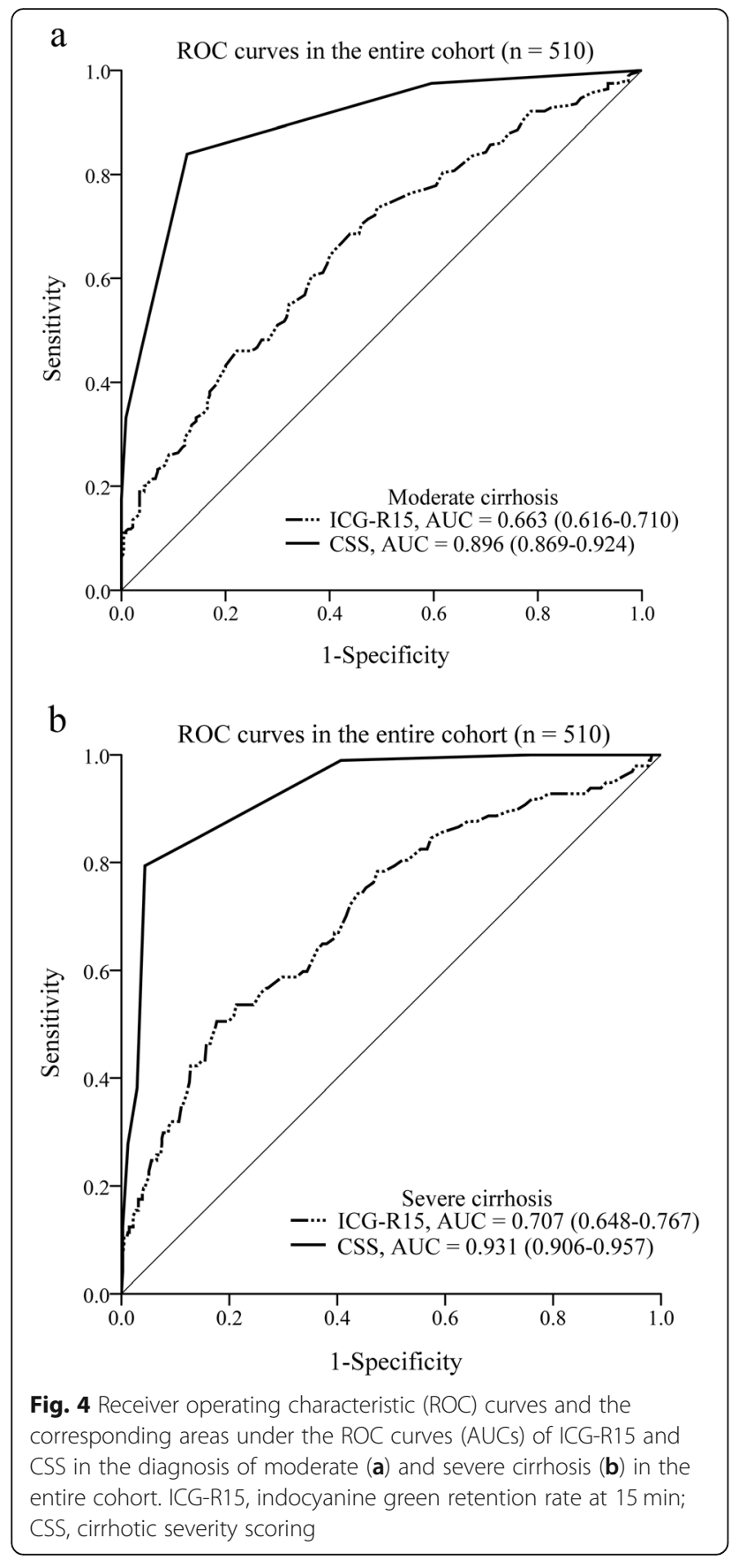

single-center, and only patients with Child-Pugh grade A liver function were included in this study. Thus, further larger and multicenter studies including patients with different Child-Pugh grades are needed to validate the results of the current study.

\section{Conclusions}

There was only a mild relationship between ICG-R15 and the pathological severity of liver cirrhosis in HCC with Child-Pugh grade A liver function, and the majority of
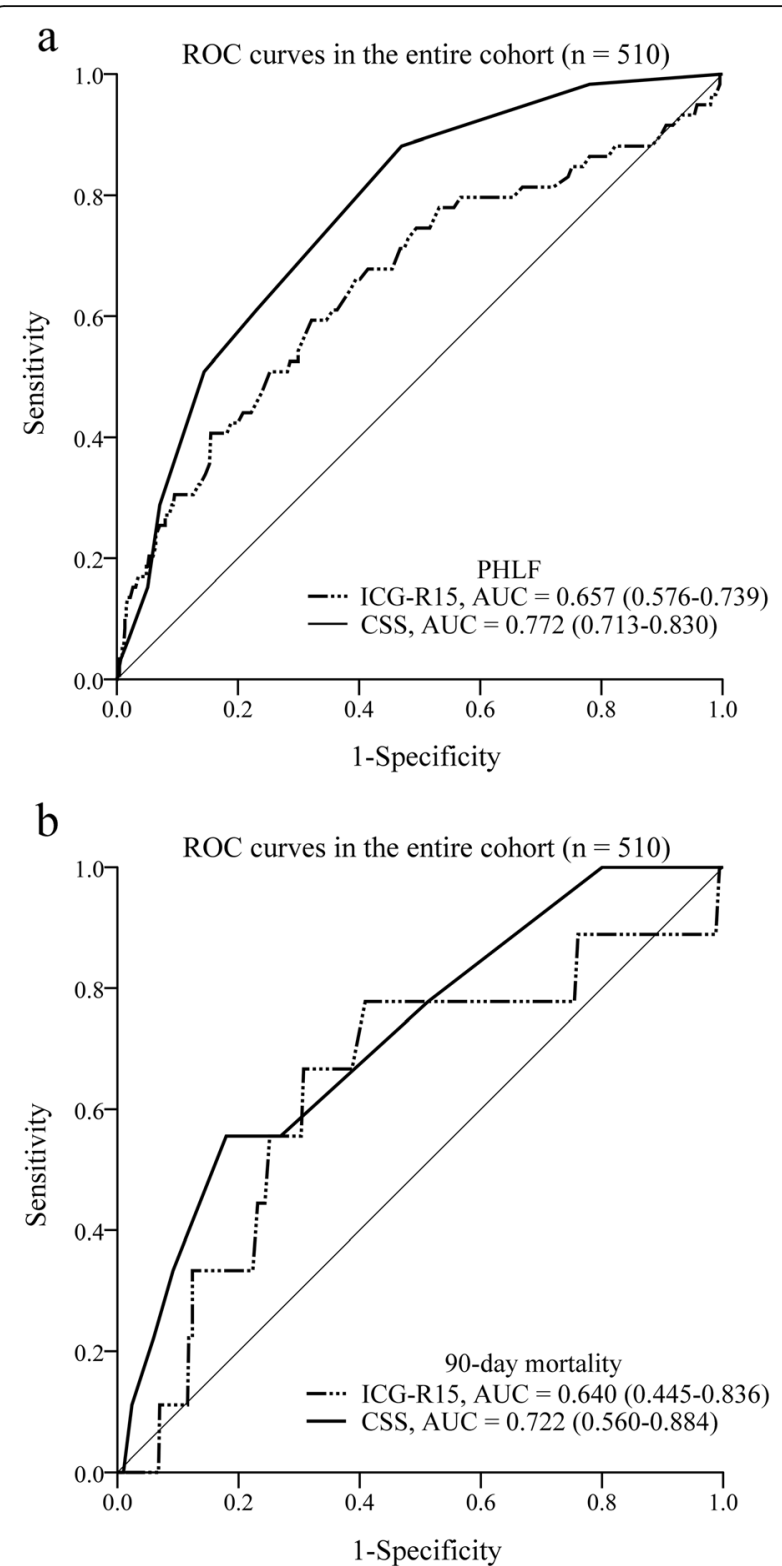

Fig. 5 Receiver operating characteristic (ROC) curves and the corresponding areas under the ROC curves (AUCs) of ICG-R15 and CSS in predicting PHLF (a) and 90-day mortality (b) in the entire cohort. ICG-R15, indocyanine green retention rate at $15 \mathrm{~min}$; CSS, cirrhotic severity scoring; PHLF, posthepatectomy liver failure patients in various degrees of liver cirrhosis had a normal ICG-R15 value. CSS was more effective than ICG-R15 in evaluating cirrhotic severity and still functioned well in patients with ICG-R15 in the normal range. In addition, CSS was superior to ICG-R15 in predicting PHLF and 90-day mortality. More studies are needed to further validate CSS in patients with different Child-Pugh grades.

\section{Abbreviations}

HCC: Hepatocellular carcinoma; CSS: Cirrhotic severity scoring; ICG-

R15: Indocyanine green retention rate at 15 min; PHLF: Posthepatectomy 
liver failure; ROC: Receiver operating characteristic; AUC: Area under the ROC curve; APRI: Aspartate aminotransferase-platelet ratio index; FIB-4: Fibrosis index based on the four factors; TE: Transient elastography; H\&E: Hematoxylin-eosin; ALT: Alanine aminotransferase; AST: Aspartate aminotransferase; INR: International normalized ratio; PT: Prothrombin time; RBC: Red blood cell

\section{Acknowledgements}

The authors thank Dr. Cong Liu and Dr. Yaobing Chen, Department of Pathology, Tongji Hospital, Huazhong University of Science and Technology, China, for their help in the pathological study.

\section{Authors' contributions}

Zhiyong Huang and Xiaoping Chen contributed to the study design. Jin Gu, Binyong Liang, and Zunyi Zhang contributed to the collection of data. Jin Gu and Erlei Zhang contributed to the analysis and interpretation of data. Jin Gu and Zhiyong Huang contributed to the writing of the article. Zhiyong Huang and Erlei Zhang contributed to the financial support. Zhiyong Huang, Xiaoping Chen, and Erlei Zhang contributed to the revision of the article and statistical analysis. All authors read and approved the final version of the article.

\section{Funding}

This work was supported by funding from the National Major Science and Technology Project of China (no. 2017ZX10203207-002-005) to Prof. Zhiyong Huang and funding from the National Natural Science Foundation of China (no. 81902839) to Dr. Erlei Zhang.

\section{Availability of data and materials}

The datasets used and analyzed during the current study are available from the corresponding author on reasonable request.

\section{Ethics approval and consent to participate}

This study was conducted in accordance with the standards of the Declaration of Helsinki and approved by the medical ethics committee of Tongji Hospital, Huazhong University of Science and Technology, China. Written informed consent for hepatectomy and further research was obtained from all patients.

\section{Consent for publication}

Not applicable.

\section{Competing interests}

The authors declare that they have no competing interests.

Received: 27 November 2019 Accepted: 13 April 2020

Published online: 23 April 2020

\section{References}

1. Bray F, Ferlay J, Soerjomataram I, Siegel RL, Torre LA, Jemal A. Global cancer statistics 2018: GLOBOCAN estimates of incidence and mortality worldwide for 36 cancers in 185 countries. CA Cancer J Clin. 2018;68:394-424.

2. Bruix J, Gores GJ, Mazzaferro V. Hepatocellular carcinoma: clinical frontiers and perspectives. Gut. 2014;63:844-55

3. Chen XP, Wu ZD, Huang ZY, Qiu FZ. Use of hepatectomy and splenectomy to treat hepatocellular carcinoma with cirrhotic hypersplenism. Br J Surg 2005;92:334-9.

4. Rahbari NN, Garden OJ, Padbury R, Brooke-Smith M, Crawford M, Adam R, et al. Posthepatectomy liver failure: a definition and grading by the international study group of liver surgery (ISGLS). Surgery. 2011;149:713-24.

5. van Mierlo KM, Schaap FG, Dejong CH, Olde Damink SW. Liver resection for cancer: new developments in prediction, prevention and management of postresectional liver failure. J Hepatol. 2016;65:1217-31.

6. Zhou SJ, Zhang EL, Liang BY, Zhang ZY, Dong KS, Hou P, et al. Morphologic severity of cirrhosis determines the extent of liver resection in patients with hepatocellular carcinoma and Child-Pugh grade A cirrhosis. J Surg Res. 2016;200:444-51

7. Seyama Y, Kokudo N. Assessment of liver function for safe hepatic resection. Hepatol Res. 2009;39:107-16.
8. Halle BM, Poulsen TD, Pedersen HP. Indocyanine green plasma disappearance rate as dynamic liver function test in critically ill patients. Acta Anaesthesiol Scand. 2014;58:1214-9.

9. Faybik P, Krenn CG, Baker A, Lahner D, Berlakovich G, Steltzer H, et al. Comparison of invasive and noninvasive measurement of plasma disappearance rate of indocyanine green in patients undergoing liver transplantation: a prospective investigator-blinded study. Liver Transpl. 2004;10:1060-4.

10. Schneider PD. Preoperative assessment of liver function. Surg Clin North Am. 2004:84:355-73.

11. Imamura H, Sano K, Sugawara Y, Kokudo N, Makuuchi M. Assessment of hepatic reserve for indication of hepatic resection: decision tree incorporating indocyanine green test. J Hepatobiliary Pancreat Surg. 2005;12:16-22.

12. Cadranel JF, Rufat P, Degos F. Practices of liver biopsy in France: results of a prospective nationwide survey. For the group of epidemiology of the French association for the study of the liver (AFEF). Hepatology. 2000;32: 477-81.

13. Bedossa $P$, Dargere $D$, Paradis V. Sampling variability of liver fibrosis in chronic hepatitis C. Hepatology. 2003;38:1449-57.

14. Zhang EL, Zhang ZY, Wang SP, Xiao ZY, Gu J, Xiong M, et al. Predicting the severity of liver cirrhosis through clinical parameters. J Surg Res. 2016;204: 274-81.

15. Jensen DM. Endoscopic screening for varices in cirrhosis: findings, implications, and outcomes. Gastroenterology. 2002;122:1620-30.

16. Pol B, Campan P, Hardwigsen J, Botti G, Pons J, Le Treut YP. Morbidity of major hepatic resections: a 100-case prospective study. Eur J Surg. 1999;165: 446-53.

17. Kim MY, Cho MY, Baik SK, Park HJ, Jeon HK, Im CK, et al. Histological subclassification of cirrhosis using the Laennec fibrosis scoring system correlates with clinical stage and grade of portal hypertension. J Hepatol. 2011;55:1004-9.

18. DeLong ER, DeLong DM, Clarke-Pearson DL. Comparing the areas under two or more correlated receiver operating characteristic curves: a nonparametric approach. Biometrics. 1988:44:837-45.

19. Makuuchi M, Kosuge T, Takayama T, Yamazaki S, Kakazu T, Miyagawa S, et al. Surgery for small liver cancers. Semin Surg Oncol. 1993;9:298-304.

20. Ishikawa M, Yogita S, Miyake H, Fukuda Y, Harada M, Wada D, et al. Clarification of risk factors for hepatectomy in patients with hepatocellular carcinoma. Hepato Gastroenterol. 2002;49:1625-31.

21. Moller S, la Cour SE, Madsen JL, Bendtsen F. Indocyanine green retention test in cirrhosis and portal hypertension: accuracy and relation to severity of disease. J Gastroenterol Hepatol. 2019;34:1093-9.

22. Lau H, Man K, Fan ST, Yu MC, Lo CM, Wong J. Evaluation of preoperative hepatic function in patients with hepatocellular carcinoma undergoing hepatectomy. Br J Surg. 1997:84:1255-9.

23. Wai CT, Greenson JK, Fontana RJ, Kalbfleisch JD, Marrero JA, Conjeevaram HS, et al. A simple noninvasive index can predict both significant fibrosis and cirrhosis in patients with chronic hepatitis C. Hepatology. 2003;38:518-26.

24. Vallet-Pichard A, Mallet V, Nalpas B, Verkarre V, Nalpas A, Dhalluin-Venier V, et al. FIB-4: an inexpensive and accurate marker of fibrosis in HCV infection. comparison with liver biopsy and fibrotest. Hepatology. 2007:46:32-6.

25. Cross TJ, Rizzi P, Berry PA, Bruce M, Portmann B, Harrison PM. King's score: an accurate marker of cirrhosis in chronic hepatitis C. Eur J Gastroenterol Hepatol. 2009;21:730-8.

26. Ziol M, Handra-Luca A, Kettaneh A, Christidis C, Mal F, Kazemi F, et al. Noninvasive assessment of liver fibrosis by measurement of stiffness in patients with chronic hepatitis C. Hepatology. 2005;41:48-54.

\section{Publisher's Note}

Springer Nature remains neutral with regard to jurisdictional claims in published maps and institutional affiliations. 